\title{
Remarks on general zeroth-order Randić and general sum-connectivity indices
}

\author{
M. M. Matejić, P. D. Milošević, E. I. Milovanović, I. Ž. Milovanović
}

\begin{abstract}
Let $G=(V, E), V=\left\{v_{1}, v_{2}, \ldots, v_{n}\right\}$, be a simple connected graph with $n$ vertices, $m$ edges and vertex degree sequence $d_{1} \geq d_{2} \geq \cdots \geq d_{n}>0, d_{i}=d\left(v_{i}\right)$. General zeroth-order Randić index of $G$ is defined as ${ }^{0} R_{\alpha}(G)=\sum_{i=1}^{n} d_{i}^{\alpha}$, and general sum-connectivity index as $\chi_{\alpha}(G)=\sum_{i \sim j}\left(d_{i}+d_{j}\right)^{\alpha}$, where $\alpha$ is an arbitrary real number. In this paper we establish a relationship between ${ }^{0} R_{\alpha+\beta}(G),{ }^{0} R_{\alpha-\beta}(G)$ and ${ }^{0} R_{\alpha}(G)$, as well as $\chi_{\alpha+\beta}(G), \chi_{\alpha-\beta}(G)$ and $\chi_{\alpha}(G)$, where $\alpha$ and $\beta$ are arbitrary real numbers. By the appropriate choice of parameters $\alpha$ and $\beta$, a number of new/old inequalities that reveal relationships between various vertex and edge degree-based topological indices are obtained.
\end{abstract}

\section{Introduction}

Let $G=(V, E), V=\left\{v_{1}, v_{2}, \ldots, v_{n}\right\}, E=\left\{e_{1}, e_{2}, \ldots, e_{m}\right\}$, be a simple connected graph with $n$ vertices, $m$ edges and $\Delta=d_{1} \geq d_{2} \geq \cdots \geq d_{n}=\delta>0, d_{i}=d\left(v_{i}\right)$, and $\Delta_{e}=d\left(e_{1}\right)+2 \geq$ $d\left(e_{2}\right)+2 \geq \cdots \geq d\left(e_{m}\right)+2=\delta_{e}$ be sequences of vertex and edge degrees in $G$, respectively. With $i \sim j$ we denote adjacency of vertices $v_{i}$ and $v_{j}$ in $G$. As usual, $L(G)$ denotes a line graph of $G$.

A graph invariant, or topological index, is a numeric quantity associated with a graph which characterize the topology of graph and is invariant under graph automorphism. Gutman and Trinajstić in [9] introduced a vertex-degree-based topological index, later named the first Zagreb index, $M_{1}(G)$, as

$$
M_{1}(G)=\sum_{i=1}^{n} d_{i}^{2}
$$

In [13] degree 2 in the previous expression is replaced with an arbitrary real number $\alpha$, i.e. generalization of the first Zagreb index is introduced, known as the general zeroth-order

Manuscript received July 20, 2018; accepted February 15, 2019.

M. M. Matejić, P. D. Milošević, E. I. Milovanović, I. Ž. Milovanović are with the Faculty of Electronic Engineering, Beogradska 14, P.O.Box 73, 18000 Niš, Serbia 
Randić index as

$$
{ }^{0} R_{\alpha}(G)=\sum_{i=1}^{n} d_{i}^{\alpha} .
$$

It is also met under names general first Zagreb index [16] and variable first Zagreb index [22].

In addition to the aforementioned first Zagreb index $M_{1}(G)={ }^{0} R_{2}(G)$, in this paper we are interested in the following special cases of invariant ${ }^{0} R_{\alpha}(G):{ }^{m} M_{1}(G)={ }^{0} R_{-2}(G)$ (the modified first Zagreb index [28]), $I D(G)={ }^{0} R_{-1}(G)$ (the inverse degree or modified total adjacency index [5] and [28]), ${ }^{0} R(G)={ }^{0} R_{-1 / 2}(G)$ (zeroth-order Randić index), $F(G)=$ ${ }^{0} R_{3}(G)$ (forgotten topological index [8]). More on these topological indices can be found in $[1,3,10,17,20]$ and in the references cited therein.

As shown in $[22,28]$, the first Zagreb index can be also expressed as

$$
M_{1}(G)=\sum_{i \sim j}\left(d_{i}+d_{j}\right) .
$$

The sum-connectivity index, $\operatorname{SCI}(G)$, is defined as [35]

$$
S C I(G)=\sum_{i \sim j}\left(d_{i}+d_{j}\right)^{-1 / 2} .
$$

A generalization of the previous two degree-based graph invariant was introduced in [36], and named general sum-connectivity index, $\chi_{\alpha}(G)$. It is defined as

$$
\chi_{\alpha}(G)=\sum_{i \sim j}\left(d_{i}+d_{j}\right)^{\alpha}
$$

where $\alpha$ is an arbitrary real number.

Let $e=\left\{v_{i}, v_{j}\right\}$ be an arbitrary edge of graph $G$. Then $d(e)=d_{i}+d_{j}-2$, and consequently we have

$$
\chi_{\alpha}(G)=\sum_{i=1}^{m}\left(d\left(e_{i}\right)+2\right)^{\alpha} .
$$

Therefore $\chi_{\alpha}(G)$ is also edge-degree-based topological index. In this paper we are interested in the following special cases of this topological index: ${ }^{m} H M(G)=\chi_{-2}(G)$ (the modified hyper-Zagreb index), $H(G)=2 \chi_{-1}(G)$ (the harmonic index [5]), $H M(G)=\chi_{2}(G)$ (the hyper-Zagreb index [33]).

One can easily see that

$$
H M(G)=F(G)+2 M_{2}(G)
$$

where

$$
M_{2}(G)=\sum_{i \sim j} d_{i} d_{j}
$$


is the second Zagreb index defined in [11].

In this paper we prove inequalities that establish relations between topological indices ${ }^{0} R_{\alpha+\beta}(G),{ }^{0} R_{\alpha-\beta}(G)$ and ${ }^{0} R_{\alpha}(G)$, as well as $\chi_{\alpha+\beta}(G), \chi_{\alpha-\beta}(G)$ and $\chi_{\alpha}(G)$, where $\alpha$ and $\beta$ are arbitrary real numbers. In special cases, for the appropriate choice of parameters $\alpha$ and $\beta$, we obtain a number of old/new inequalities for different vertex and edge degreebased topological indices.

\section{Preliminaries}

In this section we recall one discrete analytical inequality for real number sequences that will be used in the paper.

Let $p=\left(p_{i}\right)$ be nonnegative and $x=\left(x_{i}\right)$ be positive real number sequences with the properties $p_{1}+p_{2}+\cdots+p_{n}=1$ and $0<r \leq x_{i} \leq R<+\infty, i=1,2, \ldots, n$. Then [29] (see also [27])

$$
\sum_{i=1}^{n} p_{i} x_{i}+r R \sum_{i=1}^{n} \frac{p_{i}}{x_{i}} \leq r+R
$$

with equality if and only if $x_{i} \in\{r, R\}$ for every $i=1,2, \ldots, n$.

\section{Relations between ${ }^{0} R_{\alpha+\beta}(G),{ }^{0} R_{\alpha-\beta}(G)$ and ${ }^{0} R_{\alpha}(G)$}

In the following theorem we establish relations between graph invariants ${ }^{0} R_{\alpha+\beta}(G),{ }^{0} R_{\alpha-\beta}(G)$ and ${ }^{0} R_{\alpha}(G)$.

Theorem 1. Let $G$ be a simple connected graph with $n \geq 2$ vertices. Then for any real numbers $\alpha$ and $\beta$ hold

$$
{ }^{0} R_{\alpha+\beta}(G)-\left(\Delta^{\beta}+\delta^{\beta}\right){ }^{0} R_{\alpha}(G)+(\Delta \delta){ }^{\beta}{ }^{0} R_{\alpha-\beta}(G) \leq 0 .
$$

Equality holds if and only if $\beta=0$ or $d_{i} \in\{\delta, \Delta\}$ for every $i=1,2, \ldots, n$.

Proof. Let $\beta \geq 0$. For $p_{i}=\frac{d_{i}^{\alpha}}{\sum_{i=1}^{n} d_{i}^{\alpha}}, x_{i}=d_{i}^{\beta}, i=1,2, \ldots, n, r=\delta^{\beta}$ and $R=\Delta^{\beta}$, the inequality (1) transforms into

$$
\frac{\sum_{i=1}^{n} d_{i}^{\alpha+\beta}}{\sum_{i=1}^{n} d_{i}^{\alpha}}+(\Delta \delta)^{\beta} \frac{\sum_{i=1}^{n} d_{i}^{\alpha-\beta}}{\sum_{i=1}^{n} d_{i}^{\alpha}} \leq \Delta^{\beta}+\delta^{\beta},
$$

i.e.

$$
\frac{{ }^{0} R_{\alpha+\beta}(G)}{{ }^{0} R_{\alpha}(G)}+(\Delta \delta)^{\beta} \frac{{ }^{0} R_{\alpha-\beta}(G)}{{ }^{0} R_{\alpha}(G)} \leq \Delta^{\beta}+\delta^{\beta},
$$


wherefrom (2) is obtained.

In a similar way the inequality (2) is obtained when $\beta \leq 0$.

Equality in (3), i.e. in (2), holds if and only if $\beta=0$ or $d_{i} \in\{\delta, \Delta\}$ for every $i=$ $1,2, \ldots, n$.

Corollary 1. Let $G$ be a simple connected graph with $n \geq 2$ vertices. Then for any real numbers $\alpha$ and $\beta$ hold

$$
{ }^{0} R_{\alpha-\beta}(G){ }^{0} R_{\alpha+\beta}(G) \leq \frac{{ }^{0} R_{\alpha}(G)^{2}}{4}\left(\sqrt{\frac{\Delta^{\beta}}{\delta^{\beta}}}+\sqrt{\frac{\delta^{\beta}}{\Delta^{\beta}}}\right)^{2}
$$

Equality holds if and only if $\beta=0$ or $G$ is a regular graph.

Proof. By applying the arithmetic-geometric mean inequality for real numbers (see e.g. [27]) to the following inequality

$$
{ }^{0} R_{\alpha+\beta}(G)+(\Delta \delta)^{\beta}{ }^{0} R_{\alpha-\beta}(G) \leq\left(\Delta^{\beta}+\delta^{\beta}\right){ }^{0} R_{\alpha}(G),
$$

we get

$$
2 \sqrt{(\Delta \delta)^{\beta}{ }^{0} R_{\alpha+\beta}(G){ }^{0} R_{\alpha-\beta}(G)} \leq\left(\Delta^{\beta}+\delta^{\beta}\right){ }^{0} R_{\alpha}(G),
$$

wherefrom (4) follows.

Corollary 2. Let $G$ be a simple connected graph with $n \geq 2$ vertices. Then for any real number $\alpha$ holds

$$
\begin{gathered}
{ }^{0} R_{\alpha+1}(G)-(\Delta+\delta){ }^{0} R_{\alpha}(G)+\Delta \delta^{0} R_{\alpha-1}(G) \leq 0, \\
{ }^{0} R_{2 \alpha}(G)-\left(\Delta^{\alpha}+\delta^{\alpha}\right){ }^{0} R_{\alpha}(G)+n(\Delta \delta)^{\alpha} \leq 0, \\
{ }^{0} R_{\alpha}(G)-n\left(\Delta^{\alpha}+\delta^{\alpha}\right)+(\Delta \delta)^{\alpha}{ }^{0} R_{-\alpha}(G) \leq 0, \\
{ }^{0} R_{\alpha+1}(G)^{0} R_{\alpha-1}(G) \leq \frac{{ }^{0} R_{\alpha}(G)^{2}}{4}\left(\sqrt{\frac{\Delta}{\delta}}+\sqrt{\frac{\delta}{\Delta}}\right)^{2}, \\
2(\Delta \delta)^{\alpha / 2} \sqrt{n^{0} R_{2 \alpha}(G)} \leq\left(\Delta^{\alpha}+\delta^{\alpha}\right){ }^{0} R_{\alpha}(G), \\
2(\Delta \delta)^{\alpha / 2} \sqrt{{ }^{0} R_{\alpha}(G){ }^{0} R_{-\alpha}(G)} \leq n\left(\Delta^{\alpha}+\delta^{\alpha}\right) .
\end{gathered}
$$

Remark 1. The inequalities (5) and (7) were proven in [23], whereas (6) and (8) in [30]. 
Corollary 3. Let $G$ be a simple connected graph with $n \geq 2$ vertices and $m$ edges. Then

$$
\begin{gathered}
M_{1}(G) \leq 2 m(\Delta+\delta)-n \Delta \delta, \\
M_{1}(G) \leq \frac{m^{2}}{n}\left(\sqrt{\frac{\Delta}{\delta}}+\sqrt{\frac{\delta}{\Delta}}\right)^{2}, \\
F(G) \leq 2 m\left(\Delta^{2}+\delta^{2}\right)-I D(G)(\Delta \delta)^{2}, \\
F(G) \leq(\Delta+\delta) M_{1}(G)-2 m \delta \Delta, \\
F(G) \leq \frac{M_{1}(G)^{2}}{8 m}\left(\sqrt{\frac{\Delta}{\delta}}+\sqrt{\frac{\delta}{\Delta}}\right)^{2}, \\
I D(G) \leq \frac{n(\Delta+\delta)-2 m}{\Delta \delta}, \\
m_{M_{1}}(G) \leq \frac{(\Delta+\delta) I D(G)-n}{\Delta \delta}, \\
M_{2}(G) \leq \frac{1}{2}(\Delta+\delta) M_{1}(G)-m \Delta \delta, \\
M_{1}(G) \leq \frac{I D(G)^{2}}{4 n}\left(\sqrt{\frac{\Delta}{\delta}}+\sqrt{\frac{\delta}{\Delta}}\right)^{2}, \\
\frac{M_{1}(G)^{2}}{16 m}\left(\sqrt{\frac{\Delta}{\delta}}+\sqrt{\frac{\delta}{\Delta}}\right)^{2}, \\
M_{2}(G) \leq m\left(\Delta^{2}+\delta^{2}\right)-\frac{1}{2}(\Delta \delta)^{2} I D(G), \\
\sqrt{n I D(G)} \leq \frac{n^{2}\left(\Delta^{2}+\delta^{2}\right)^{2}}{4(\Delta \delta)^{2}}, \\
\left.\sqrt{\frac{1}{\Delta}}+\frac{1}{\sqrt{\delta}}\right){ }^{0} R(G),
\end{gathered}
$$

Remark 2. The inequality (9) was proven in [4] (see also [12, 18]), the inequality (10) in [7, 15, 19, 34], the inequality (11) in [14], and (12) in [23]. 


\section{Relations between $\chi_{\alpha+\beta}(G), \chi_{\alpha-\beta}(G)$ and $\chi_{\alpha}(G)$}

In the next theorem we prove the inequality that establishes relation between topological indices $\chi_{\alpha+\beta}(G), \chi_{\alpha-\beta}(G)$ and $\chi_{\alpha}(G)$.

Theorem 2. Let $G$ be a simple connected graph with $m \geq 2$ edges. Then for any real numbers $\alpha$ and $\beta$ hold

$$
\chi_{\alpha+\beta}(G)-\left(\Delta_{e}^{\beta}+\delta_{e}^{\beta}\right) \chi_{\alpha}(G)+\left(\Delta_{e} \delta_{e}\right)^{\beta} \chi_{\alpha-\beta}(G) \leq 0 .
$$

Equality holds if and only if $\beta=0$ or $d\left(e_{i}\right)+2 \in\left\{\delta_{e}, \Delta_{e}\right\}$ for every $i=1,2, \ldots, m$.

Proof. Let $\beta \geq 0$. For $p_{i}=\frac{\left(d\left(e_{i}\right)+2\right)^{\alpha}}{\sum_{i=1}^{m}\left(d\left(e_{i}\right)+2\right)^{\alpha}}, x_{i}=\left(d\left(e_{i}\right)+2\right)^{\beta}, i=1,2, \ldots, m, r=\delta_{e}^{\beta}$ and $R=\Delta_{e}^{\beta}$, the inequality (1) becomes

$$
\frac{\sum_{i=1}^{m}\left(d\left(e_{i}\right)+2\right)^{\alpha+\beta}}{\sum_{i=1}^{m}\left(d\left(e_{i}\right)+2\right)^{\alpha}}+\left(\Delta_{e} \delta_{e}\right)^{\beta} \frac{\sum_{i=1}^{m}\left(d\left(e_{i}\right)+2\right)^{\alpha-\beta}}{\sum_{i=1}^{m}\left(d\left(e_{i}\right)+2\right)^{\alpha}} \leq \Delta_{e}^{\beta}+\delta_{e}^{\beta},
$$

i.e.

$$
\frac{\chi_{\alpha+\beta}(G)}{\chi_{\alpha}(G)}+\left(\Delta_{e} \delta_{e}\right)^{\beta} \frac{\chi_{\alpha-\beta}(G)}{\chi_{\alpha}(G)} \leq \Delta_{e}^{\beta}+\delta_{e}^{\beta},
$$

wherefrom we get (13).

Similarly, the inequality (13) is obtained when $\beta \leq 0$.

Equality in (14), and consequently in (13), holds if and only if $\beta=0$ or $d\left(e_{i}\right)+2 \in$ $\left\{\boldsymbol{\delta}_{e}, \Delta_{e}\right\}$ for every $i=1,2, \ldots, m$.

By a similar procedure as in case of Corollary 1, the following corollary of Theorem 2 is proved.

Corollary 4. Let $G$ be a simple connected graph with $m \geq 2$ edges. Then for any real numbers $\alpha$ and $\beta$ hold

$$
\chi_{\alpha+\beta}(G) \chi_{\alpha-\beta}(G) \leq \frac{\chi_{\alpha}(G)^{2}}{4}\left(\sqrt{\frac{\Delta_{e}^{\beta}}{\delta_{e}^{\beta}}}+\sqrt{\frac{\delta_{e}^{\beta}}{\Delta_{e}^{\beta}}}\right)^{2} .
$$

Equality holds if and only if $\beta=0$ or $L(G)$ is a regular graph. 
Corollary 5. Let $G$ be a simple connected graph with $m \geq 2$ edges. Then for any real number $\alpha$ holds

$$
\begin{gathered}
\chi_{\alpha+1}(G)-\left(\Delta_{e}+\delta_{e}\right) \chi_{\alpha}(G)+\Delta_{e} \delta_{e} \chi_{\alpha-1}(G) \leq 0, \\
\chi_{2 \alpha}(G)-\left(\Delta_{e}^{\alpha}+\delta_{e}^{\alpha}\right) \chi_{\alpha}(G)+m\left(\Delta_{e} \delta_{e}\right)^{\alpha} \leq 0, \\
\chi_{\alpha}(G)-m\left(\Delta_{e}^{\alpha}+\delta_{e}^{\alpha}\right)+\left(\Delta_{e} \delta_{e}\right)^{\alpha} \chi_{-\alpha}(G) \leq 0, \\
\chi_{\alpha+1}(G) \chi_{\alpha-1}(G) \leq \frac{\chi_{\alpha}(G)^{2}}{4}\left(\sqrt{\frac{\Delta_{e}}{\delta_{e}}}+\sqrt{\frac{\delta_{e}}{\Delta_{e}}}\right)^{2}, \\
2\left(\Delta_{e} \delta_{e}\right)^{\alpha / 2} \sqrt{m \chi_{2 \alpha}(G)} \leq\left(\Delta_{e}^{\alpha}+\delta_{e}^{\alpha}\right) \chi_{\alpha}(G), \\
2\left(\Delta_{e} \delta_{e}\right)^{\alpha / 2} \sqrt{\chi_{\alpha}(G) \chi_{-\alpha}(G)} \leq m\left(\Delta_{e}^{\alpha}+\delta_{e}^{\alpha}\right) .
\end{gathered}
$$

Remark 3. The inequalities (15) and (16) were proven in [24]. The inequality (17) is stronger than inequality

$$
2(\Delta \delta)^{\alpha / 2} \sqrt{m \chi_{2 \alpha}(G)} \leq\left(\Delta^{\alpha}+\delta^{\alpha}\right) \chi_{\alpha}(G)
$$

proven in [31].

Corollary 6. Let $G$ be a simple connected graph with $m \geq 2$ edges. Then

$$
\begin{gathered}
{ }^{m} H M(G) \leq \frac{1}{\Delta_{e} \delta_{e}}\left(\frac{\left(\Delta_{e}+\delta_{e}\right) H(G)}{2}-m\right), \\
{ }^{m} H M(G) \leq \frac{1}{\left(\Delta_{e} \delta_{e}\right)^{2}}\left(\Delta_{e}^{2}+\delta_{e}^{2}-H M(G)\right), \\
{ }^{m} H M(G) \leq \frac{H(G)^{2}}{16 m}\left(\sqrt{\frac{\Delta_{e}}{\delta_{e}}}+\sqrt{\frac{\delta_{e}}{\Delta_{e}}}\right)^{2} \\
{ }^{m} H M(G) H M(G) \leq \frac{m^{2}\left(\Delta_{e}^{2}+\delta_{e}^{2}\right)^{2}}{4\left(\Delta_{e} \delta_{e}\right)^{2}} \\
{ }^{m}(G) \leq \frac{2}{\Delta_{e} \delta_{e}}\left(m\left(\Delta_{e}+\delta_{e}\right)-M_{1}(G)\right) \\
M_{1}(G) H(G) \leq m^{2}\left(\sqrt{\frac{\Delta_{e}}{\delta_{e}}}+\sqrt{\frac{\delta_{e}}{\Delta_{e}}}\right)^{2} \\
H M(G) \leq\left(\Delta_{e}+\delta_{e}\right) M_{1}(G)-m \Delta_{e} \delta_{e}
\end{gathered}
$$




$$
\begin{gathered}
H M(G) \leq \frac{M_{1}(G)^{2}}{4 m}\left(\sqrt{\frac{\Delta_{e}}{\delta_{e}}}+\sqrt{\frac{\delta_{e}}{\Delta_{e}}}\right)^{2}, \\
S C I(G) \geq \frac{\left(\Delta_{e} \delta_{e}\right)^{1 / 4}}{\sqrt{\Delta_{e}}+\sqrt{\delta_{e}}} \sqrt{2 m H(G)} .
\end{gathered}
$$

Remark 4. The inequalities (19) and (20) were proven in [25], and in special cases in [24] and [21]. More about this type of inequalities can be found in [26].

The inequality (18) is stronger than inequality

$$
2 M_{1}(G) H(G) \leq m^{2}\left(\sqrt{\frac{\Delta}{\delta}}+\sqrt{\frac{\delta}{\Delta}}\right)^{2},
$$

which was proven in [32].

\section{Acknowledgement}

This work was supported by the Serbian Ministry for Education, Science and Technological development, grants TR32009 and TR32012.

\section{References}

[1] A. Ali, I. Gutman, E. Milovanović, I. Milovanović, Sum of powers of the degrees of graphs: extremal results and bounds, MATCH Commun. Math. Comput. Chem., 80 (2018), $5-84$.

[2] A. Ali, L. Zhong, I. Gutman, Harmonic index and its generalizations: extremal results and bounds, MATCH Commun. Math. Comput. Chem., 81 (2019), 249-311.

[3] B. Borovićanin, K. Ch. Das, B. Furtula, I. Gutman, Bounds for Zagreb indices, MATCH Commun. Math. Comput. Chem., 78 (2017), 17-100.

[4] K. C. DAS, Maximizing the sum of the squares of the degrees of a graph, Discr. Math., 285 (2004), 57-66.

[5] S. FajtlowicZ, On conjectures of Graffiti-II, Congr. Numer., 60 (1987), 187-197.

[6] F. Falahati-Nezhad, M. Azari, Bounds on the hyper-Zagreb index, J. Appl. Math. Inform., 34 (2016), 319-330.

[7] G. H. FATH-TABAR, Old and new Zagreb indices of graphs, MATCH Commun. Math. Comput. Chem., 65 (2011), 79-84.

[8] B. Furtula, I. Gutman, A forgotten topological index, J. Math. Chem., 53 (2015), 11841190.

[9] I. Gutman, N. TRinajstić, Graph theory and molecular orbitals. Total $\pi$-electron energy of alternant hydrocarbons, Chem. Phys. Lett., 17 (1972), 535-538. 
[10] I. Gutman, E. Milovanović, I. Milovanović, Beyond the Zagreb indices, AKCE Int. J. Graph Combin. (in press)

[11] I. Gutman, B. Ruščić, N. Trinajstić, C. F. Wilcox, Graph theory and molecular orbitals. XII. Acyclic polyenes, J. Chem. Phys., 62 (1975), 3399-3405.

[12] S. Hosamani, B. BASAVANAgOud, New upper bounds for the first Zagreb index, MATCH Commun. Math. Comput. Chem., 74 (2015), 97-101.

[13] Y. HU, X. LI, Y. SHI, T. XU, I. GUTMAN, On molecular graphs with smallest and greatest zeroth-order general Randić index, MATCH Commun. Math. Comput. Chem., 54 (2005), 425-434.

[14] A. ILIĆ, B. ZHOU, On reformulated Zagreb indices, Discr. Appl. Math., 160 (2012), 204-209.

[15] A. ILIĆ, M. ILIĆ, B. LiU, On the upper bounds for the first Zagreb index, Kragujevac J. Math., 35 (2011), 173-182.

[16] X. LI, J. ZHENG, A unified approach to the extremal trees for different indices, MATCH Commun. Math. Comput. Chem., 54 (2005), 195-208.

[17] X. LI, I. Gutman, Mathematical aspects of Randić type molecular structure descriptors, Univ. Kragujevac, Kragujevac, 2006.

[18] J. LI, W. C. Shiu, A. ChAng, On the Laplacian Estrada index of a graph, Appl. Anal. Discr. Math., 3 (2009), 147-156.

[19] M. LIU, B. LIU, New sharp upper bounds for the first Zagreb index, MATCH Commun. Math. Comput. Chem., 62 (2009), 689-698.

[20] Y. Ma, S. CaO, Y. Shi, I. Gutman, M. Dehmer, B. Furtula, From the connectivity index to various Randić-type descriptors, MATCH Commun. Math. Comput. Chem., 80 (2018), 85-106.

[21] M. M. Matejić, I. Ž. Milovanović, E. I. Milovanović, On bounds for harmonic topological index, Filomat, 32(1) (2018), 311-317.

[22] A. Miličević, S. Nikolić, On variable Zagreb indices, Croat. Chem. Acta, 77 (2004), $97-101$.

[23] I. Ž. Milovanović, V. M. Ćirić, I. Z. Milentijević, E. I. Milovanović, On some spectral, vertex and edge degree-based graph invariants, MATCH Commun. Math. Comput. Chem., 77 (2017), 177-188.

[24] I. Ž. Milovanović, E. I. Milovanović, M. Matejić, Some inequalities for general sumconnectivity index, MATCH Commun. Math. Comput. Chem., 79 (2018), 477-489.

[25] I. Ž. Milovanović, E. I. Milovanović, I. Gutman, B. Furtula, Some inequalities for the forgotten topological index, Int. J. Appl. Graph Theory, 1(1) (2017), 1-15.

[26] E. Milovanović, M. Matejić, I. Milovanović, Some new upper bounds for the hyperZagreb index, Discrete Math. Lett., 1 (2019), 30-35.

[27] D. S. Mitrinović, P. M. VAsić, Analytic inequalities, Springer Verlag, Berlin-HeidelbergNew York, 1970.

[28] S. Nikolić, G. KovačEvić, A. MiličEvić, N. Trinajstić, The Zagreb indices 30 years after, Croat. Chem. Acta, 76 (2003), 113-124. 
[29] B. C. Rennie, On a class of inequalities, J. Austral. Math. Soc., 3 (1963), 442-448.

[30] J. M. Rodriguez, J. L. Sanchez, J. M. Sigarreta, CMMSE-on the first general Zagreb index, J. Math. Chem., 56 (2018), 1849-1864.

[31] J. M. Rodriguez, J. M. Sigarreta, New results on the harmonic index and its generalizations, MATCH Commun. Math. Comput. Chem., 78 (2017), 387-404.

[32] J. M. Rodriguez, J. M. Sigarreta, Harmonic index, In: Bounds in chemical graph theory-Basics (I. Gutman, B. Furtula, K. C. Das, E. Milovanović, I. Milovanović, Eds.), Univ. Kragujevac, Kragujevac, 2017, pp. 229-281.

[33] G. H. ShiRdel, H. RezAPOUR, A. M. SAYADI, The hyper-Zagreb index of graph operations, Iranian J. Math. Chem., 4 (2013), 213-220.

[34] G. Tian, T. HuAng, S. CUi, Bounds on the algebraic connectivity of graphs, Adv. Math., 41(2) (2012), 217-224.

[35] B. Zhou, N. Trinajstić, On a novel connectivity index, J. Math. Chem., 46 (2009), $1252-$ 1270.

[36] B. Zhou, N. Trinajstić, On general sum-connectivity index, J. Math. Chem., 47 (2010), 210-218. 